\title{
Main rotor-body action for virtual blades model
}

\author{
Alexander Kusyumov ${ }^{1, a}$, Sergey Kusyumov ${ }^{2}$, Sergey Mikhailov ${ }^{3}$, Elena Romanova ${ }^{4}$, Konstantin Phayzullin ${ }^{5}$, \\ Evgeny Lopatin ${ }^{6}$ and G. Barakos ${ }^{7}$ \\ ${ }^{1-6}$ Kazan National Research Technical University n.a. A. N. Tupolev (KNRTU-KAI), Kazan, Russia \\ ${ }^{7}$ University of Glasgow, Glasgow, UK
}

\begin{abstract}
This research aims to investigate a virtual blade model and assess rotor influence on helicopter fuselage aerodynamics. The rotor disk is discretized in the azimuthal direction, and a time-varied pressure jump is applied in regions occupied by the blades. To obtain the pressure jump, an actuator disk is employed using uniform and non-uniform blade load distribution, based on momentum theory.
\end{abstract}

\section{Introduction}

Rotor Computational Fluid Dynamics (CFD) calculations are challenging. The complexity of the problem is due to the flow unsteadiness, the coupled aerodynamics and aeroelasticity of rotor blades, and the presence of vortical effects and wakes characterized by a range of flow scales, both laminar and turbulent. One can also include the large variety of flight conditions. Despite these difficulties, CFD methods now are becoming mature enough to simulate the flow characteristics around helicopters [1,2].

Computations with detailed resolution of the helicopter blades are still expensive. For this reason, actuator models of rotors and propellers have their place in the tool-chain of engineering methods available to helicopter designers. An advantage of the actuator disk model is its simplicity, efficiency and relatively good accuracy. Classic actuator disk models allow the use of steadystate CFD computation, significantly reducing the required computer time and memory. The actuator disk approximates the rotor using an infinitely thin source of momentum. This is modeled as a pressure jump across the disk.

The momentum source is modeled independently of the fuselage without any coupling between the two. For this reason, the effect of the rotor disk is simplified and the method, although computationally efficient, can only be used for initial estimates of the fuselage loads [3, 4]. The other peculiarity of this formulation is the steadystate formulation that allows for time averaged estimates of rotor body interactions. More advanced concepts of the actuator-disk methods are the regionalized actuator disk models considered in [5] for flows around propellers.

In reality, the effect of blades on the fuselage is unsteady. For this reason the vortical structure of the flow around a fuselage in rotor-body interaction is different than what is predicted by steady-state actuatordisk methods. This problem can be partly solved using

Corresponding author: postbox7@mail.ru the unsteady actuator disk model considered in [5] for flows around propellers. The propeller disk surface is divided along azimuthally direction, and time-varied pressure jump and tangential velocity in some regions are specified. The flow parameters are updated with respect to the azimuth angle at each time step to simulate the unsteady flow effect induced by the rotating blades.

The aim of the present paper is to simulate the flow around a helicopter rotor/fuselage case in forward flight mode using the Virtual Blade Actuator (VBA) model instead of resolved blades. To obtain the pressure jump for the mean rotor thrust the uniform actuator disk was modeled using uniform and non-uniform blade load distribution, based on momentum theory.

Rotor action changes the aerodynamic performance of fuselage and the properties of the flow (including turbulence modeling parameters) around the helicopter. The parameters of turbulence determine the broadband noise of the flow [6].

\section{Computational Case}

A CAD model of a helicopter fuselage corresponding to an early design of the ANSAT-P model of the modern, light-medium ANSAT helicopter (produced by the JSC Kazan Helicopters) is used. The shape of the ANSAT-P model is similar to the prototype of the ANSAT helicopter (Figure 1) used for preliminary studies in the T-1K wind tunnel of Kazan National Research Technical University named after A.N. Tupolev (KNRTU-KAI). The aerodynamic performance for this model was first obtained for a "blind" CFD modeling exercise [7].

Some geometric parameters of the model are presented in Table 1.

Figure 2 shows the CAD model of the fuselage with the actuator disk and the virtual blades. In this paper a sector of the virtual blades is used to simplify the computational procedure (the area of the virtual blades corresponds to the planform area of the resolved blades). 
The location of virtual blades varies in time according to the location of resolved blades.

Table 1. CAD model parameters

\begin{tabular}{|c|c|}
\hline Parameter & Value \\
\hline Number of blades $N$ & 4 \\
\hline Radius of rotor $R, \mathrm{~m}$ & 0.575 \\
\hline Chord $c, \mathrm{~m}$ & 0.032 \\
\hline Root radius $R_{0}, \mathrm{~m}$ & 0.144 \\
\hline Length of fuselage $L_{F}, \mathrm{~m}$ & 1.1 \\
\hline
\end{tabular}

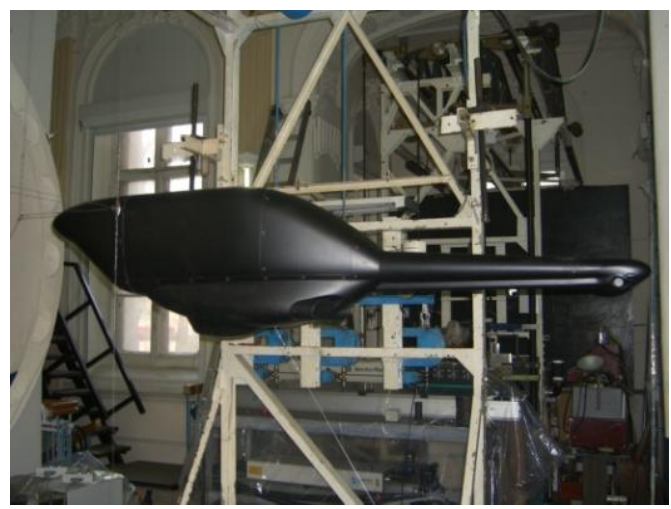

Fig. 1. ANSAT-P fuselage model in the test section

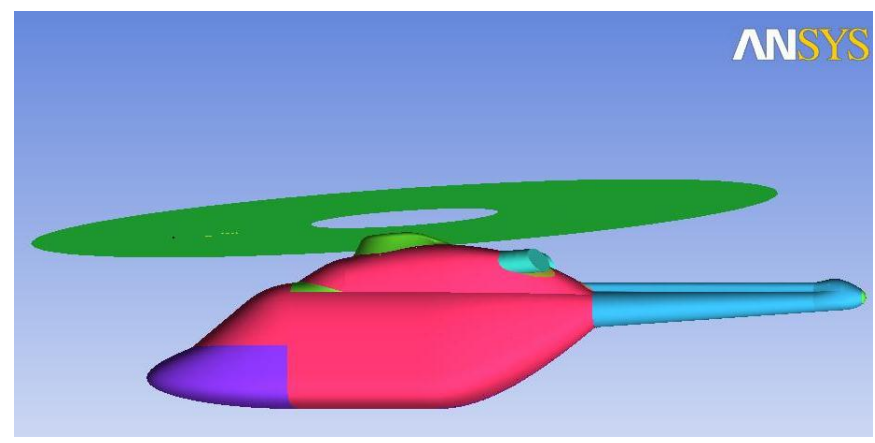

a)

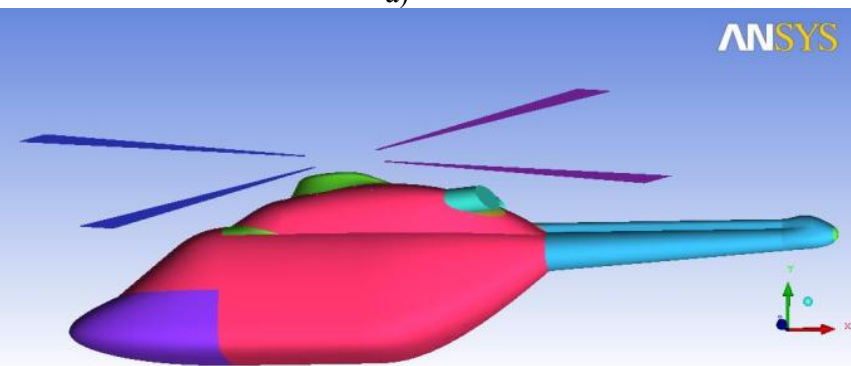

b)

Fig. 2. CAD model image of fuselage with the actuator disk (a) and the virtual blades (b)

\section{Numerical approach}

The simulation of the flow around the isolated fuselage ANSAT-P was conducted using the FLUENT CFD code. For modeling an unstructured tetra-grid with 4.800.000 elements was used that was built using the ANSYS ICEM program. The grid in the immediate vicinity of the body was designed to resolve the flow boundary layer and was constructed using about 20 prismatic layers.
The value of $y+$ for all turbulence models was up to 1. At least 15 elements were kept inside the boundary layer for sufficient resolution. The mesh had the first cell height of $0.005 \mathrm{~mm}$ above the wall. Other parameters of grid and the grid sensitivity question were considered in reference [7].

Results using the tetra-mesh, were compared against the the HMB code of Liverpool University that employed a multi-block hexa-mesh (11.000.000 elements).

Table 2 presents the drag $\left(C_{D}\right)$ and lift $\left(C_{L}\right)$ obtained using CFD approach for pith angle $\alpha=0$ degrees in comparison to experimental data. For the numerical simulation, the $k-\omega$ and the Spalart-Allmaras (SA) models of turbulence were used with Fluent and $k-\omega$ model was used with HMB. The conditions of the wind tunnel experiment and CFD modelling corresponded to the free stream Mach number and the Reynolds numbers were of 0.1 and $4.4 \cdot 10^{6}$, respectively.

Table 2. CFD results

\begin{tabular}{|c|c|c|c|c|}
\hline \multirow{2}{*}{$\begin{array}{l}\text { Computation } \\
\text { mode }\end{array}$} & \multicolumn{2}{|c|}{ CFD } & \multicolumn{2}{|c|}{ Experiment } \\
\hline & $C_{D}$ & $C_{L}$ & $C_{D}$ & $C_{L}$ \\
\hline Fluent, SA & 0.101 & -0.07 & \multirow{3}{*}{0.09} & \multirow{3}{*}{-0.07} \\
\hline Fluent, $k-\omega$ & 0.102 & -0.076 & & \\
\hline $\mathrm{HMB}, k-\omega$ & 0.095 & -0.075 & & \\
\hline
\end{tabular}

As the VBA model of actuator requires a transient formulation the Spalart-Allmaras turbulence model was chosen for computations to minimize time of simulation.

\section{Flow conditions}

The actuator disk approximates the rotor using an infinitely thin source of momentum. This is modeled as a pressure jump across the disk. The momentum source is modeled independently of the fuselage without any feedback between the two.

To obtain the pressure jump for the mean rotor thrust the uniform actuator disk was modeled proceeding from the thrust force $T$ (corresponding to the weight of helicopter). The constant pressure jump was applied to the grid points of the actuator disk, inside a radius $R$ of $0.574 \mathrm{~m}$ (Figure 2), with a disk tilt of $\alpha_{a}=4.5$ degrees. Computations were performed for free stream velocity $V_{\infty}$ of $53 \mathrm{~m} / \mathrm{s}$, angular frequency of rotation $\omega=2 \pi f(f=50$ $\mathrm{Hz})$. For this condition the $C_{T} / \mu^{2}$ was $0.1744(\Delta p=300$ $\mathrm{Pa})$. Here $C_{T}=2 T /\left(\rho \pi R^{2} V_{t}^{2}\right)$ is the trust coefficient, $\mu=$ $V_{\infty} / V_{t}$ is the advanced ratio, $V_{t}=\omega R$ is the rotor tip velocity, $\rho$ is the air density.

For a non-uniform disk a "typical" distribution of the pressure was estimated based on references $[8,9]$. The distribution of the pressure jump on the disk surface is given by:

$$
\Delta p=\rho \gamma\left[\gamma \operatorname{sign}(\delta) / 2+V_{\infty} \cos \left(\alpha-\alpha_{a}+\delta\right)\right]
$$

(the same approach was used in [10]). Here $\delta$ is the angle of vortex cylinder slope (a small value for cruise velocity conditions), $\alpha_{a}$ is the angle of actuator disk tilt. A general expression for the function $\gamma$ of circulation distribution on the disk surface can be written as 


$$
\gamma(r, \psi)=\gamma_{r}(r)+\gamma_{s}(r) \sin (\psi),
$$

where $\psi$ is azimuth angle of considered point on disk surface. One can note here that rotor thrust value is determined basically by the $\gamma_{r}(r)$ function. The $\gamma_{s}(r)$ determines inequality of circulation distribution for advancing and retreating blades and for the present research is accepted $\gamma_{s}(r) \equiv 0$ (according to the recommendation of reference [8]).

The distribution of the averaged blade load $\gamma_{r}$ can be written in the form

$$
\gamma_{r}=C f_{r}\left(r_{n}\right)
$$

where $f_{r}\left(r_{n}\right)=r_{n}^{2}\left(2-r_{n}^{2}-r_{n}^{4}\right), \quad r_{n}=r / R \quad$ is normalized radius. The value of $C$ is determined by the formula

$$
\begin{gathered}
C=1.989 V_{\infty}\left[-\cos \left(\alpha-\alpha_{a}+\delta\right)+\right. \\
\left.+\sqrt{\cos ^{2}\left(\alpha-\alpha_{a}+\delta\right)+1.207 C_{T} / \mu^{2}}\right] .
\end{gathered}
$$

Eventually in this work it is assumed that the pressure distribution depends on the local disk radius $r$ only:

$$
\Delta p_{0}=\rho\left[\gamma_{r}^{2} / 2+\gamma_{r} V_{\infty} \cos \left(\alpha-\alpha_{a}+\delta\right)\right] .
$$

The uniform and non-uniform disk was implemented in the HMB solver and in Fluent using user-defined functions. Figure 3 presents the uniform and nonuniform actuator disk pressure distributions vs normalized rotor disk radius for the considered conditions.

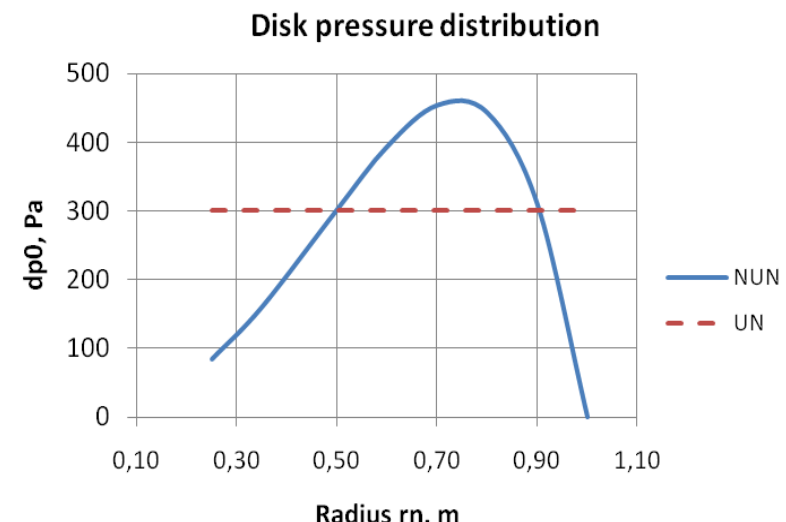

Fig. 3. Uniform and non-uniform actuator disk pressure distributions

\section{Results for classical actuator disk}

The pressure coefficient $C p=2\left(p-p_{\infty}\right) /\left(\rho V_{\infty}{ }^{2}\right)$ distribution on the fuselage surface ( $p$ is a local pressure, $p_{\infty}$ is the free stream pressure) without actuator is shown in Figure 4a (for visualization the ANSYS CFD-Post code is used). Figures $4 \mathrm{~b}$ and $4 \mathrm{c}$ show the difference $d C p=C p$ $C p_{a}$ for the uniform and nonuniform models ( $C_{p a}$ is the pressure coefficient with the actuator).

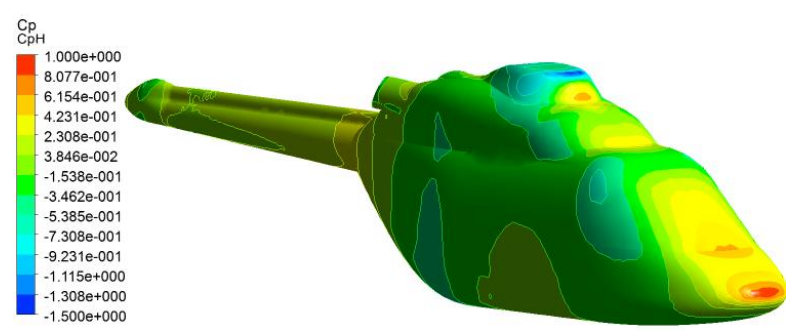

(a)

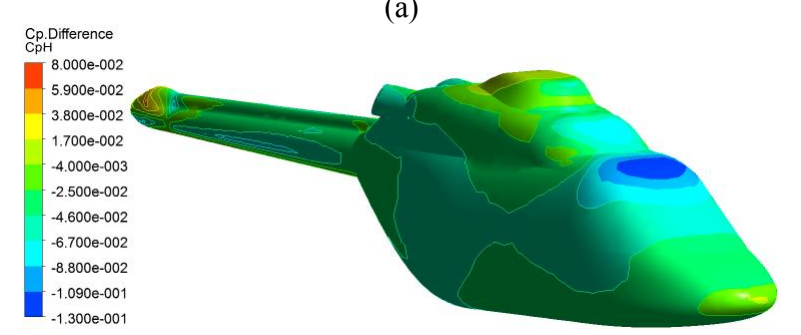

(b)

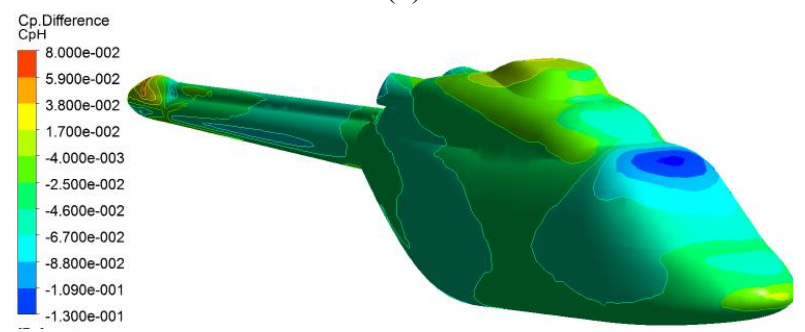

(c)

Fig 4. The pressure coefficient $C p$ distributions on the isolated fuselage surface (a), difference $d C p$ with uniform (b) and nonuniform (c) actuators

From Figure 4 it follows that the presence of the actuator disk does not lead to strong redistribution of the pressure coefficient for the considered conditions (relatively high value of the advanced ratio coefficient $\mu=0.29$ ). Some discrepancy can be observed at the front part of fuselage.

The steady-state formulation of the CFD computations allows for a simple approach (based on Proudman's formula [6]) to estimate acoustic emissions of the turbulent flow, assuming that the emitted noise is continuously distributed over a broad range of frequencies. In this case the broadband noise power, can be estimated from the RANS equations using the mean flow field, turbulent kinetic energy $k$ and the dissipation rate $\varepsilon$.

According to Proudman the total radiated acoustic power per unit volume of turbulence is:

where

$$
P_{A}=\alpha_{\varepsilon} \rho \varepsilon M_{t}^{5},
$$

$$
M_{t}=(2 k)^{1 / 2} / a \text {. }
$$

Here $a$ is the speed of sound, $\rho$ is the air density, $M_{t}$ is the turbulent Mach number. The re-scaled coefficient, $\alpha_{\varepsilon}$, is set to 0.1 .

Fluent has already implemented Proudman's formula for acoustic power per unit of volume, generated by isotropic turbulence without mean flow. As the Proudman's approach requires determination of the turbulent kinetic energy $k$ in the present paper the broadband noise is considered for $k$ - $\omega$ model.

A 2D distribution of the Sound Pressure Level (SPL, $\mathrm{dB}$ ) is given in a across the flow, located close to the junction of the tail boom and fuselage (Figure 5). 


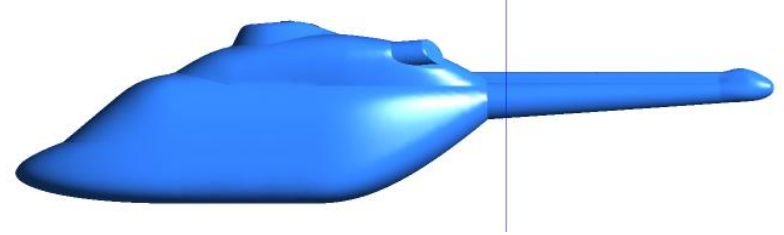

Fig.5. 2D section for SPL estimation

Figure 6a shows the 2D $\mathrm{SPL}_{0}$ distribution for the isolated fuselage with and without actuator at 0 degrees of pitch angle. Figures $6 \mathrm{~b}$ and $6 \mathrm{c}$ show the difference $d \mathrm{SPL}=\mathrm{SPL}_{0}-\mathrm{SPL}_{a}$ for the uniform and nonuniform classical actuator models $\left(\mathrm{SPL}_{a}\right.$ is the result with the actuator)

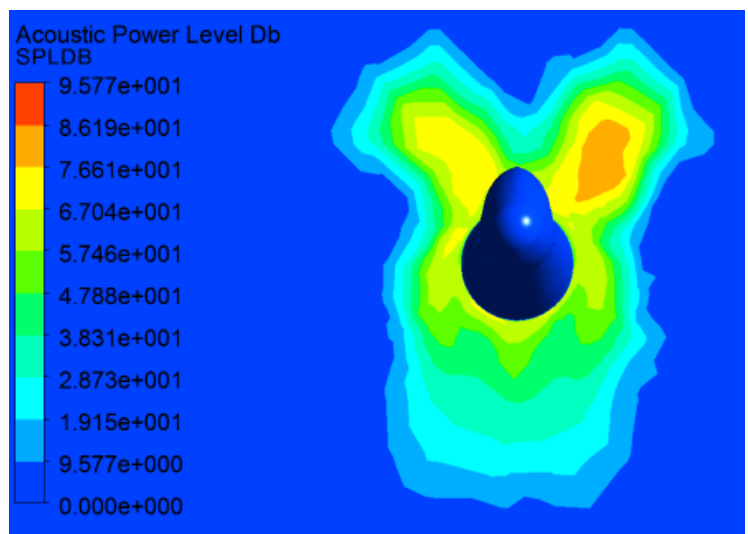

(a)

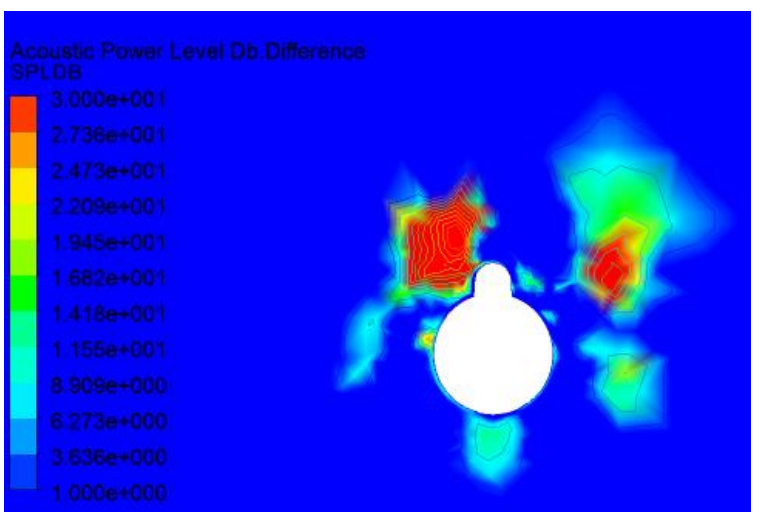

(b)

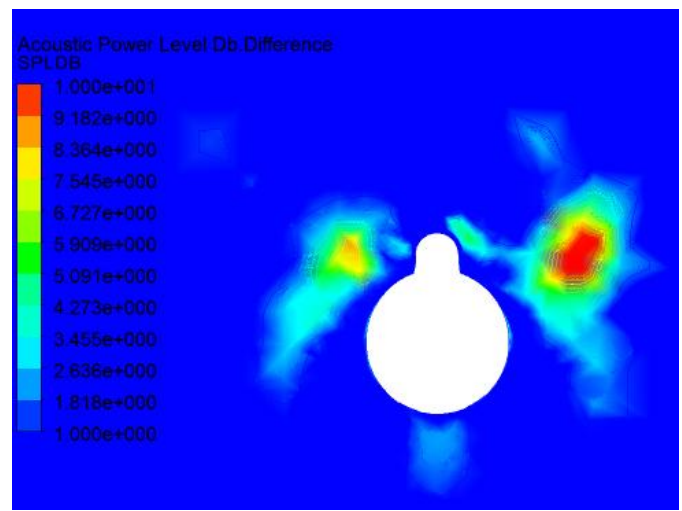

(c)

Fig.6. $2 \mathrm{D} \mathrm{SPL}_{0}$ distribution for the isolated fuselage (a) and $d$ SPL for the fuselage with uniform (b) and nonuniform (c) actuator
The uniform load distribution suggests a strong influence of the actuator on the turbulent flow properties in comparison to the nonuniform load distribution.

\section{Results for VBA model}

For the uniform and nonuniform VBA disk models simulations were conducted in unsteady mode. The simulations are described in section 4 and time a step of $\Delta t=0.0001 \mathrm{sec}$ was used corresponding to an azimuth angle step of 1.8 degrees.

Figures 7,8 present the pressure coefficient distribution at the actuator disc area and on the fuselage surface for two VBA models: uniform and nonuniform. Figure 8 shows $d C p=C p_{V B A}-C p_{a}$ between the surface pressure coefficient values. Here $C p_{a}$ is the pressure coefficient for the classical actuator disk model and $C p_{V B A}$ is the pressure coefficient for the VBA actuator disk models (uniform and nonuniform). The Lift and Drag coefficients time behavior for the uniform and nonuniform actuator disk models are presented in Figure 9. The location of the virtual blades in Figures 7,8 corresponds approximately to time $t_{1}$ (Figures $7 \mathrm{a}, 7 \mathrm{~b}, 8 \mathrm{a}$, $8 \mathrm{~b}$ ) and $t_{2}$ (Figures $7 \mathrm{c}, 7 \mathrm{~d}, 8 \mathrm{c}, 8 \mathrm{~d}$ ), so that $t_{2}-t_{1}=0.002 \mathrm{~s}$. Points 1 and 2 in Figure 9 also correspond to $t_{1}$ and $t_{2}$, respectively.
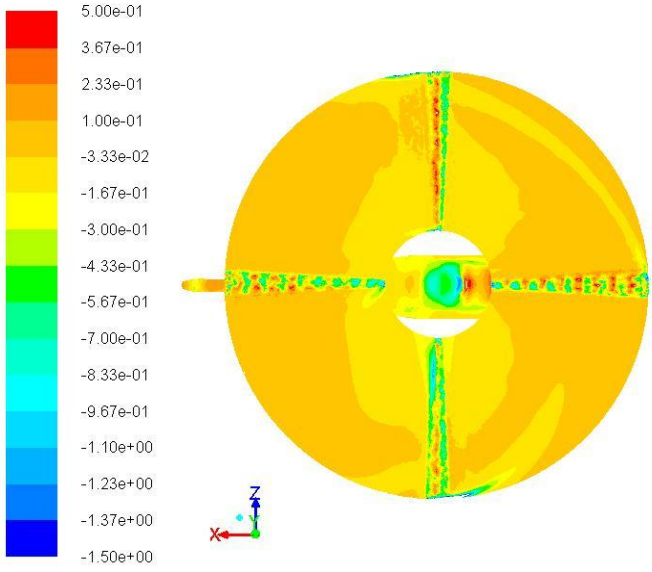

(a)
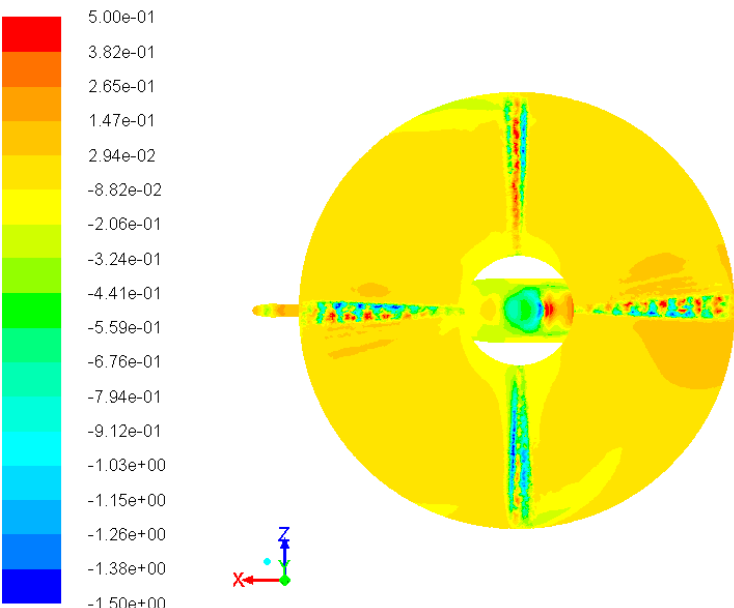

(b) 


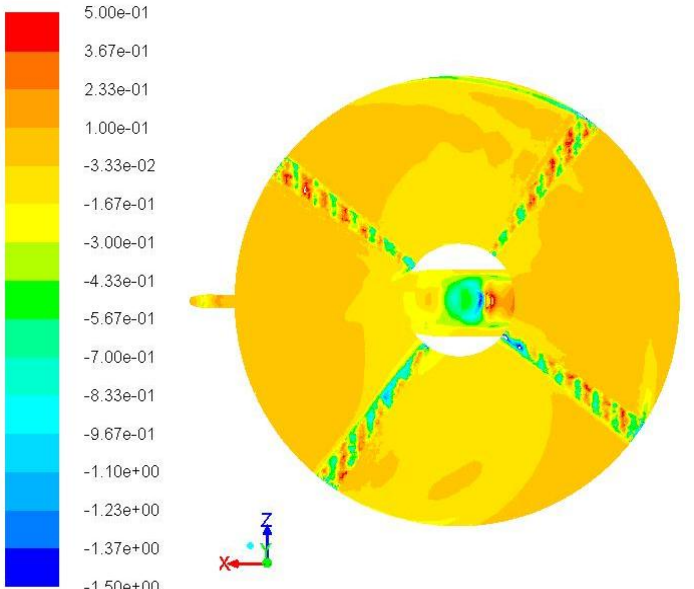

(c)
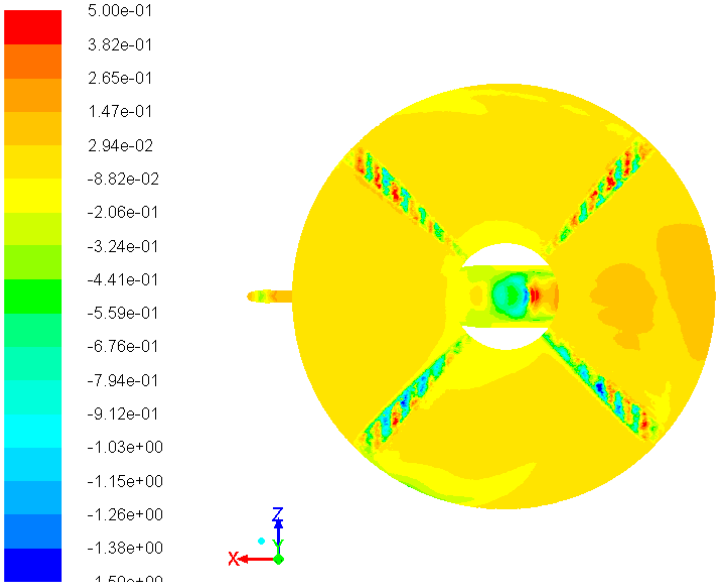

(d)

Fig.7. Distribution of the disk pressure for the uniform (a, c) and nonuniform $(\mathrm{b}, \mathrm{d})$ VBA models

For both locations of the rotor blades (presented in Figure 7) it can be seen that there is some discrepancy of the pressure coefficient near the tips of blades. Figure 8 shows minor difference between the pressure coefficients on the top front part of the fuselage surface. These discrepancies suggest differences between the values of the lift $\left(C_{L}\right)$ and $\operatorname{drag}\left(C_{D}\right)$ fuselage coefficients for the uniform and nonuniform VBA model (Figure 9).
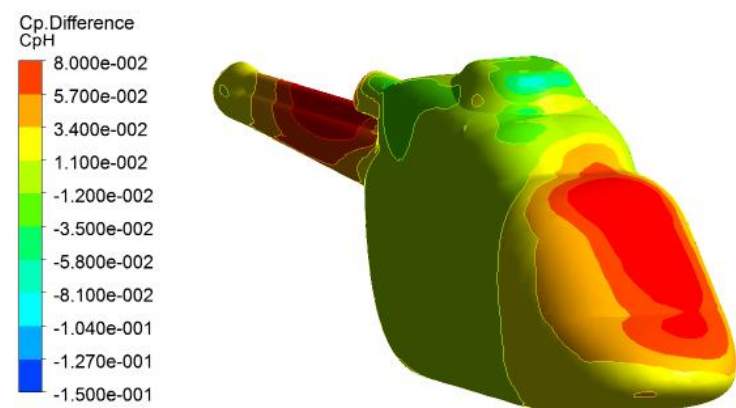

(a)
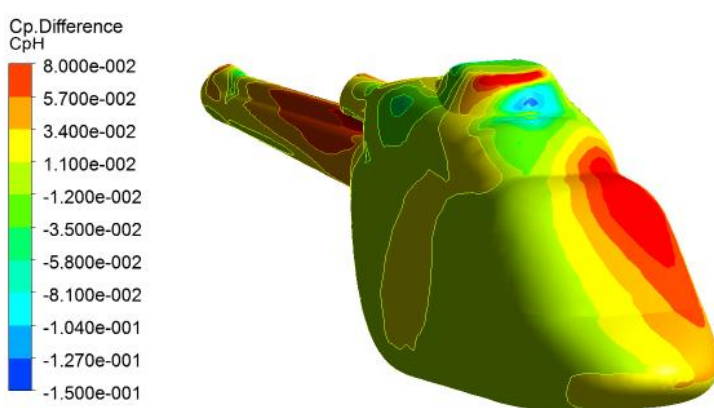

(b)
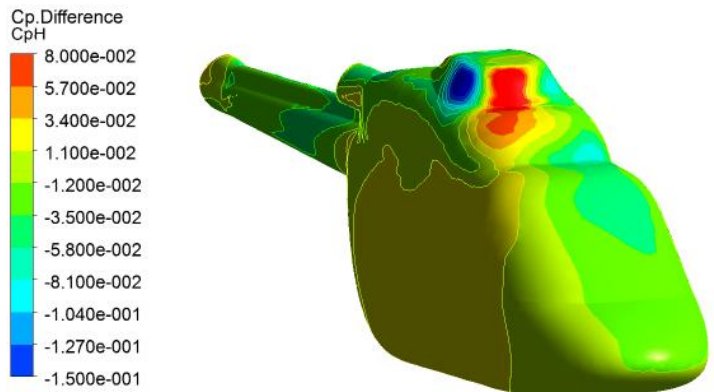

(c)
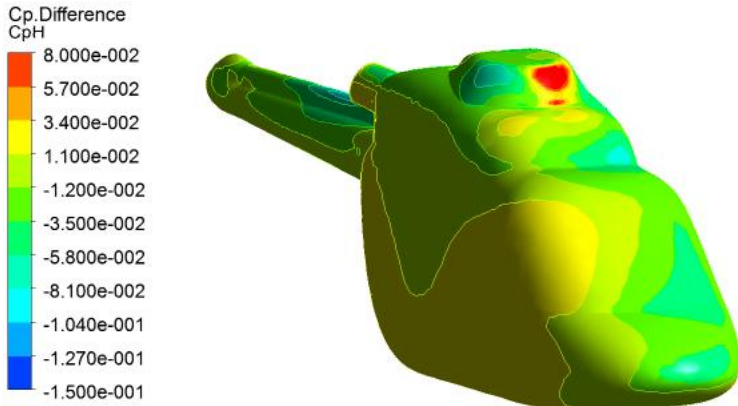

(d)

Fig.8. Distribution of the fuselage pressure for the uniform (a, c) and nonuniform (b, d) VBA models

Enhanced positive $\Delta C p$ area on the top front part of the fuselage surface for the nonuniform VBA disk model (in comparison to uniform VBA model) leads to lower $C_{L}$ peak values $\left(t_{1}\right.$ instant). For the same reason (different pressure distribution) $C_{D}$ values at $t_{2}$ instant for the uniform VBA disk model are higher in comparison to the nonuniform VBA model.

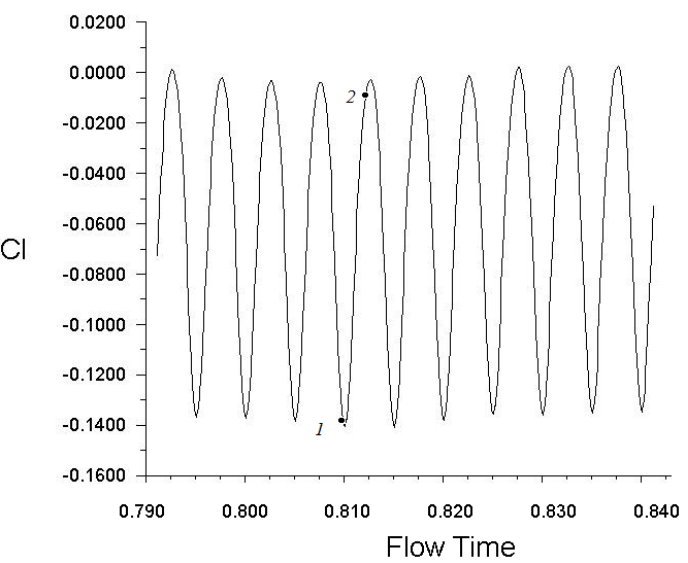


(a)

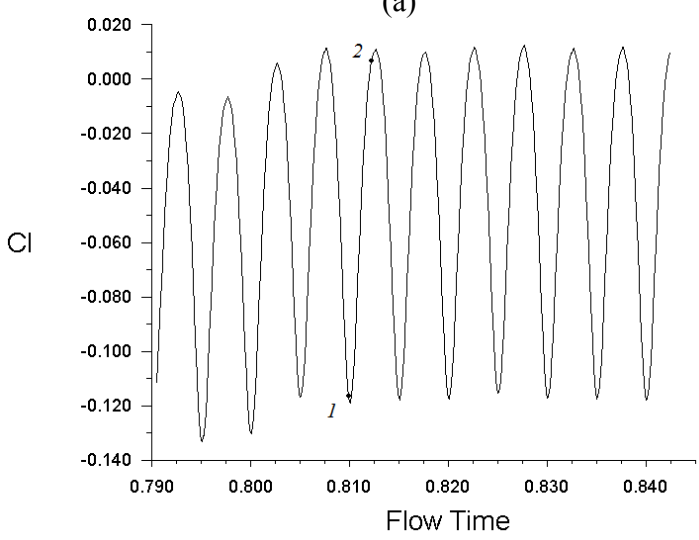

(b)

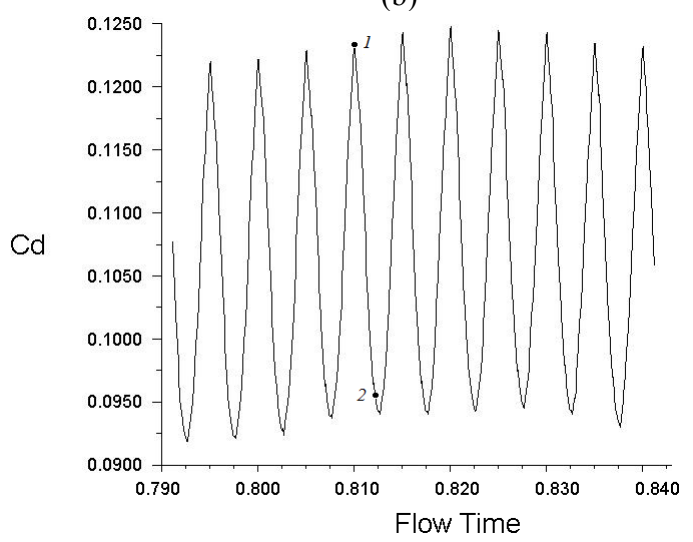

(c)

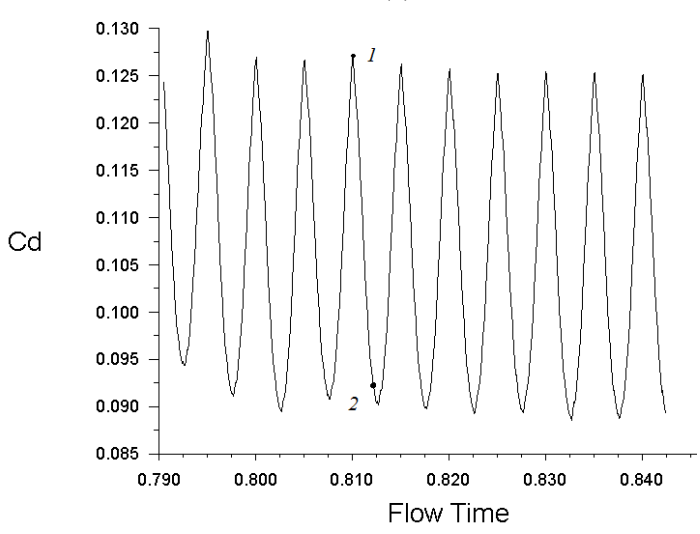

(d)

Fig.9. Lift and Drag coefficients for the uniform $(a, c)$ and nonuniform $(b, d)$ VBA models

For both uniform and nonuniform VBA disk models averaged values of lift and drag coefficients correspond to the aerodynamical coefficients for the considered steady simulation mode: $C_{L}=-0.07, C_{D}=0.101$.

\section{Conclusion}

The structure and the properties of the flow around an idealized fuselage of the ANSAT helicopter prototype were examined. The influence of the rotor on the fuselage aerodynamics was modeled with the classical disk model and the virtual blades. Uniform and nonuniform actuator load distributions were used to reproduce the main rotor flow properties. The acoustic properties (broadband noise) of the flow and the frequency range of the vortical structures were estimated using Proudman's approach and the RANS equations, in terms of mean flow field, turbulent kinetic energy and the dissipation rate.

In the future, the results of virtual blades will be compared to resolved blade data.

\section{Acknowledgments}

The support of this work via the "State tasks of the Education and Science Ministry of Russian Federation" grant (No 9.1577.2017/P $\quad \mathrm{CH}$ ) is gratefully acknowledged.

\section{References}

1. R. Steij1, and G. N. Barakos, Aerospace Science and Technology, 19, 1 (2012).

2. A. F. Antoniadis et al., Aerospace Science and Technology, 19, 1 (2012).

3. Ph. Beaumier, J.-M. Bousquet, 24-th ICAS congress (2004).

4. T. Renaud, M. D. O’Brien, M. Smith, M. Potsdam, American Helicopter Society 60th Annual Forum, (2004).

5. Q. Wang, Z. Jiang and Q. Zhang, Engineering Applications of Computational Fluid Mechanics, 8, 1 (2014).

6. I. Proudman, Proc. Roy. Soc., 214 (1952).

7. A. Kusyumov, S. Mikhailov, A. Garipov, E. Nikolaev, G. Barakos, Transactions on Control and Mechanical Systems, 1, 7 (2012).

8. H. Heyson, S. Katzoff, NACA TR, 1319 (1957).

9. V.I. Shaidakov, Proceedings of Moscow Aviation Institute, 31. (1978). [in Russian]

10. A. Kusuymov, S. Mikhailov, E. Nikolaev, N. Shilova, A. Garipov, Proceedings of the ASME 2011 International Mechanical Engineering Congress \& Exposition IMECE 2011, (2011) 